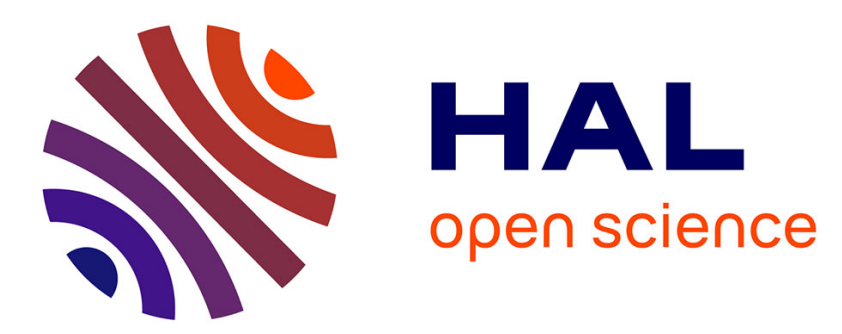

\title{
Obstacle Avoidance and Trajectory Replanification for a Group of Communicating Vehicles
}

\author{
Pierre Avanzini, Benoit Thuilot, Philippe Martinet
}

\section{To cite this version:}

Pierre Avanzini, Benoit Thuilot, Philippe Martinet. Obstacle Avoidance and Trajectory Replanification for a Group of Communicating Vehicles. ITST 2009 - 9th International Conference on ITS Telecommunications, Oct 2009, Lille, France. pp.267-272, 10.1109/ITST.2009.5399345 . hal02468620

\section{HAL Id: hal-02468620 \\ https://hal.inria.fr/hal-02468620}

Submitted on 6 Feb 2020

HAL is a multi-disciplinary open access archive for the deposit and dissemination of scientific research documents, whether they are published or not. The documents may come from teaching and research institutions in France or abroad, or from public or private research centers.
L'archive ouverte pluridisciplinaire HAL, est destinée au dépôt et à la diffusion de documents scientifiques de niveau recherche, publiés ou non, émanant des établissements d'enseignement et de recherche français ou étrangers, des laboratoires publics ou privés. 


\title{
Obstacle Avoidance and Trajectory Replanification for a Group of Communicating Vehicles
}

\author{
Pierre AVANZINI, Benoît THUILOT and Philippe MARTINET \\ LASMEA - 24 avenue des Landais - 63177 Aubière - FRANCE \\ Email: Firsname.LASTNAME@lasmea.univ-bpclermont.fr
}

\begin{abstract}
Various "Urban Transportation Systems" are currently in developing, in order to put forward solutions to congestion and pollution in dense areas. Autonomous electric vehicles in free-access can be seen as an attractive approach, in view of the large flexibility that can be expected. One instrumental functionality linked to this solution is platoon motion: several autonomous vehicles accurately follow a trajectory defined on a dedicated circulation lane, with pre-specified inter-distances. A global decentralized platoon control strategy, supported by inter-vehicle communications and relying on nonlinear control techniques is here proposed. In the nominal case, each vehicle is controlled with respect to the same smooth reference trajectory modelled by cubic B-Splines. This trajectory is locally modified and relayed on-line when a vehicle detects an obstacle. The trajectory distortion naturally reflects a driver's behaviour, since it consists in approximated clothoidal trajectories and the use of B-Splines ensures consistent connections. Experimental results, carried out with several urban vehicles, demonstrate the capabilities of the proposed approach.

Index Terms-mobile robots, nonlinear control, platooning,
\end{abstract} automatic guided vehicles, vehicle-to-vehicle communications.

\section{INTRODUCTION}

In this paper, congestion of vehicle traffic in dense areas and correlated pollution and waste of time, are addressed. Specifically, alternative transport systems, based on autonomous electric vehicles in free-access moving on dedicated lanes, are here considered, in view of the large flexibility that can be expected. One functionality of special interest is automated platooning, i.e. several autonomous vehicles following the same trajectory with pre-specified inter-distances. Such a functionality, on the one hand allows to easily adapt the transport offer to the actual need (via platoon length), and on the other hand provides more coherent motions and an enhancement in safety. Platooning is therefore considered in this paper.

Different approaches can be proposed. They can be classified into two categories, according to the information used for vehicle control: local and global strategies. The most standard approaches rely on local strategies, i.e. each vehicle is controlled exclusively from data relative to the neighboring vehicles. The well-known leader-follower approach, considering the preceding (or following) vehicle is widely used. For instance, visual tracking has been proposed in [1] and generic control laws have been designed in [2] and [3]. Alternatively, neighboring vehicles (and not only the preceding one) are taken into account when using virtual structure approaches: a structural analogy, characterized by a serial chain of springdamper, is for instance proposed in [4] and a control law is then derived from the combined front and rear virtual forces.

These strategies present however some drawbacks, the most concerning one being error accumulation: the servoing errors, induced by sensor noises and/or actuator delays, are inevitably growing from the first vehicle to the last one, leading to unacceptable oscillations. Such problems can be overcome by considering global strategies, i.e. each vehicle is now controlled from the data received from all vehicles, collected from appropriate communication channels. Most of the virtual structure approaches belong to this category. In [5], a mechanical analogy is used to design feedback controllers to achieve straight line motion. A single virtual rigid structure is also considered in [6], relying on graph theory. Nevertheless, since these techniques aim at imposing some prespecified geometric pattern, the vehicles cannot all reproduce the trajectory of the first one: in a curve, if vehicles are kept according to a straight line pattern, they all describe a distinct trajectory. In contrast, in previous work [7], a trajectorybased global control strategy has been proposed: relying on nonlinear control techniques, vehicle lateral and longitudinal control have been exactly decoupled. Lateral guidance of each vehicle with respect to the same reference path can then be achieved independently from longitudinal control, designed to maintain a pre-specified curvilinear vehicle inter-distance. Data acquisition and exchange are then predominant factors. Communication issues are therefore specifically addressed in this paper. This work is also enhanced in order that the platoon reference path can be replaned on-line if an obstacle appears ahead of the automated platoon. When vehicles move in a dedicated road infrastructure, such a problem has generally been tackled by considering lane change maneuvers [8]. The simplest solution consists in generating a trajectory by concatenating lines and arc segments [9], [10]. However, the resulting path presents then discontinuities. To overcome this difficulty, it has been proposed (e.g. [11], [12]) to consider clothoids, i.e. curves whose curvature is linearly related to the arc length, to ensure a $C^{2}$ continuous transition between the arc segments. In addition, clothoids allow to reflect a natural driver's behaviour and they are commonly used in highway route design. Since clothoidal trajectory generation requires complicated computations with Fresnel integrals, many approximation techniques [13] [14] [15] have been developed for road design. However, they are not fast enough for online path planning. On-line high-accurate representations have been proposed in [16], but the attention is only focused on the 
avoiding path pattern, neglecting the transition with respect to the original trajectory. The paper is organized as follows: the global decentralized control strategy for vehicle platooning is first sketched in Section II. Related communication issues are then adressed in Section III. The on-line trajectory replanification and generation process are then described and discussed in Section V. Finally, in Section VI, experiments carried out with three electric vehicles demonstrate the capabilities of the proposed approach.

\section{GLOBAL DECENTRALIZED CONTROL STRATEGY}

\section{A. Modeling assumptions}

Urban vehicles involved in platooning applications are supposed to move at quite low speed (less than $5 \mathrm{~m} . \mathrm{s}^{-1}$ ) on asphalted roads. Therefore dynamic effects can be neglected and a kinematic model can satisfactorily describe their behavior, as corroborated by extensive tests performed with our experimental vehicles shown in Fig. 1.

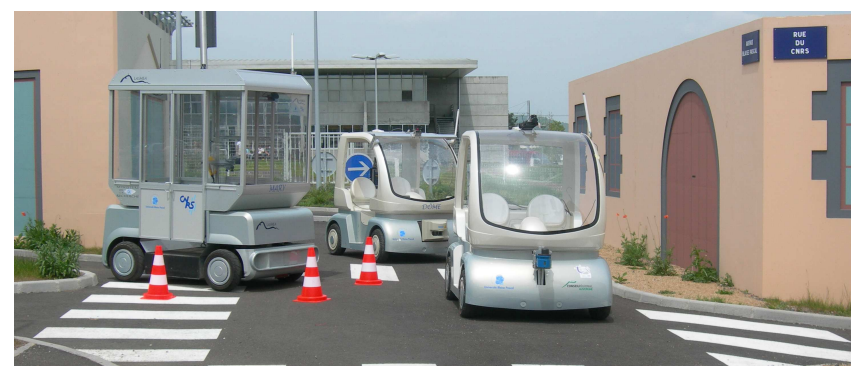

Fig. 1. Two vehicles avoiding a broken down one on PAVIN site

In this paper, the kinematic tricycle model is considered: the two actual front wheels are replaced by a unique virtual wheel located at the mid-distance between the actual wheels. The notation is illustrated in Fig. 2.

- $\Gamma$ is the common reference path for any vehicle (to be inferred from the trajectory of the first one), defined in an absolute frame $\left[A, X_{A}, Y_{A}\right]$.

- $O_{i}$ is the center of the $i^{t h}$ vehicle rear axle.

- $M_{i}$ is the closest point on $\Gamma$ to $O_{i}$.

- $s_{i}$ is the arc-length coordinate of $M_{i}$ along $\Gamma$.

- $c\left(s_{i}\right)$ is the curvature of path $\Gamma$ at $M_{i}$, and $\theta_{\Gamma}\left(s_{i}\right)$ is the orientation of the tangent to $\Gamma$ at $M_{i}$ w.r.t. $\left[A, X_{A}, Y_{A}\right]$.

- $\theta_{i}$ is the heading of $i^{\text {th }}$ vehicle w.r.t. $\left[A, X_{A}, Y_{A}\right]$.

- $\tilde{\theta}_{i}=\theta_{i}-\theta_{\Gamma}\left(s_{i}\right)$ is the angular deviation of the $i^{t h}$ vehicle w.r.t. $\Gamma$.

- $y_{i}$ is the lateral deviation of the $i^{t h}$ vehicle w.r.t. $\Gamma$.

- $\delta_{i}$ is the $i^{t h}$ vehicle front wheel steering angle.

- $L$ is the vehicle wheelbase.

- $v_{i}$ is the $i^{t h}$ vehicle linear velocity at point $O_{i}$.

\section{B. Vehicle state space model}

The configuration of the $i^{t h}$ vehicle can be described without ambiguity by the state vector $\left(s_{i}, y_{i}, \tilde{\theta}_{i}\right)$. The current values of these variables can be inferred on-line by comparing

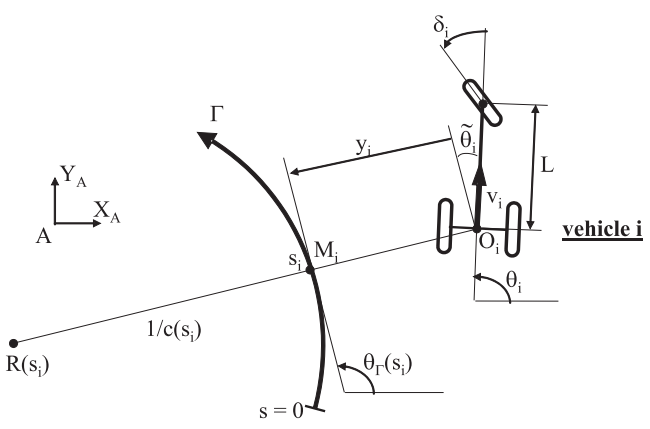

Fig. 2. Tricycle model description

vehicle absolute localization to the reference path. It can then be shown (see [17]) that tricycle state space model is:

$$
\left\{\begin{array}{l}
\dot{s}_{i}=v_{i} \frac{\cos \tilde{\theta}_{i}}{1-y_{i} c\left(s_{i}\right)} \\
\dot{y}_{i}=v_{i} \sin \tilde{\theta}_{i} \\
\dot{\tilde{\theta}}_{i}=v_{i}\left(\frac{\tan \delta_{i}}{L}-\frac{c\left(s_{i}\right) \cos \tilde{\theta}_{i}}{1-y_{i} c\left(s_{i}\right)}\right)
\end{array}\right.
$$

Platooning objectives can then be described as ensuring the convergence of $y_{i}$ and $\tilde{\theta}_{i}$ to zero, by means of $\delta_{i}$, and maintaining the gap between two successive vehicles to a fixed value $d^{*}$, by means of $v_{i}$. It is considered that $y_{i} \neq \frac{1}{c\left(s_{i}\right)}$ (i.e. vehicles are never on the reference path curvature center). In practical situations, if the $l$ vehicles are well initialized, this singularity is never encountered.

\section{Control law design}

In previous work [7], it has been shown that exact linearization techniques offer a relevant framework to address platoon control: equations (1), as most of kinematic models of mobile robots, can be converted in an exact way into a socalled chained form, see [17]. Such a conversion is attractive, since the structure of chained form equations allows to address independently lateral and longitudinal control.

Steering control laws $\delta_{i}$ can first be designed to achieve the lateral guidance of each vehicle within the platoon. In these control laws, $v_{i}$ just appears as a free parameter. Since conversion of equations (1) into chained form is exact, all nonlinearities are explicitly taken into account. High tracking performances (accurate to within $\pm 5 \mathrm{~cm}$ when relying on an RTK GPS sensor) can then be ensured, whatever initial errors or reference path curvature are. Details can be found in [18].

Control variables $v_{i}$ can then be designed to achieve longitudinal control. In nominal situation, the objective for the $i^{t h}$ vehicle is to regulate $e_{i}^{1}=s_{1}-s_{i}-(i-1) d^{*}$, i.e. the arc-length longitudinal error w.r.t. the leader. This control objective is attractive, since the location $s_{1}$ of the leader represents a common index for all the vehicles into the platoon, so that error accumulation and inherent oscillations can be avoided. In addition, since it is an arc-length error, this control objective remains consistent whatever the reference path curvature is (in contrast with euclidian inter-distances). Nevertheless, for obvious safety reasons, the location of the 
preceding vehicle cannot be ignored. Therefore, in previous work [7], the longitudinal control law has been designed to control a composite error: a smooth commutation function gives the predominance either to the global error $e_{i}^{1}$ or to the local one $e_{i}^{i-1}=s_{i-1}-s_{i}-d^{*}$ according to some security distance. Once more, exact linearization techniques have been used, so that nonlinearities in equations (1) are still explicitly accounted, ensuring high accurate regulation. More details, as well as experiment results carried out with Cycab and RobuCab vehicles (see Fig. 1), relying on RTK GPS sensors for vehicle localization and WiFi technology for inter-vehicle communications, can be found in [7].

\section{COMMUNICATION ISSUE}

The proposed global control strategy demands for intervehicle data exchanges. The $\mathrm{V} 2 \mathrm{~V}$ communication system is first presented, and then it is discussed how the asynchronous localization data that are collected, are processed in order that the control laws can rely on temporally consistent information.

\section{A. Device and communication procedure}

Inter-vehicle communications are carried out using the ieee802.11g standard, along with IPv4 and UDP protocols. A WiFi access point is located on the leader and each vehicle is connected to the wireless network via a high-level laptop equipped with an internal adapter. According to the control strategy (see Fig. 3), leader localization data are broadcasted, while other vehicles only send information to their direct follower. Data are asynchronously transferred as soon as they are supplied by the localization devices. Raw localization data consist in 97 bytes.

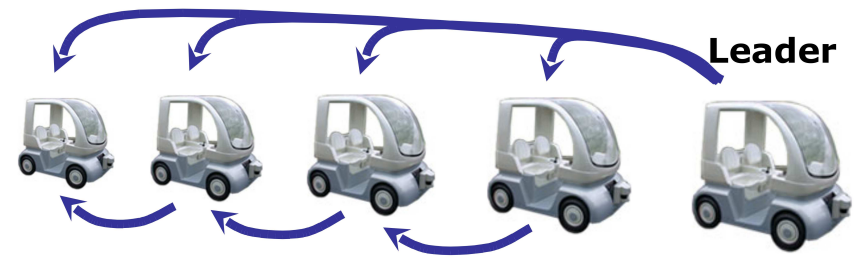

Fig. 3. Communication scheme

\section{B. Platoon information updating}

For each vehicle to be appropriately controlled, the lateral and longitudinal control laws have to be evaluated at a constant sampling frequency. However, the data from which control laws have to be inferred, are asynchronously supplied, and therefore temporally inconsistent. This difficulty appears even at the vehicle level: the vehicle state is indeed inferred from exteroceptive and proprioceptive sensors. The vehicle absolute localization is supplied by either a RTK GPS receiver or a monocular camera, while its velocity and steering angle are obtained via odometric sensors. Each sensor possesses its own latency, acquisition frequency ( $10 \mathrm{~Hz}$ with RTK GPS receivers, up to $15 \mathrm{~Hz}$ with cameras and $60 \mathrm{~Hz}$ with odometers), and possibly varying processing time (vehicle absolute localization can be derived from monocular vision with a mean image processing time equal to $30 \mathrm{~ms}$, see [19]). The platoon state, obtained by collecting other vehicle states via V2V communication, displays of course larger temporal inconsistencies: the data transmission delays have been measured within the range $[20.6 \mathrm{~ms}, 98.4 \mathrm{~ms}]$, with a $47 \mathrm{~ms}$ mean value. However, the standard troubles inherent to wireless technology, such as dropout, packet collisions or communication obstacles may occasionally increase the temporal inconsistencies between variables within the platoon state.

To appropriately control vehicles, the data to be reported into control laws should reflect the state of each vehicle within the platoon at the same time instant. To meet this demand, the software framework AROCCAM [20] is currently used. Driver modules, responsible for the communication with sensors, collect in separated threads the information. A datation procedure allows to correctly timestamp those data: the sensor latency and processing time are taken into account in order that the date reflects accurately the perception time. This date is then sent jointly with the sensor data to the other vehicles, in order for them to be able to properly use the data, whatever communication delays. To ensure that a common time reference is shared by all the vehicle computers, a simple NTP (Network Time Protocol) client allows the system clock to be set to match the time obtained when communicating with one server. It is run at a $1 \mathrm{~Hz}$ frequency on each vehicle high-level laptop, after having defined the reference server. A set of consistently dated information is then available, when AROCCAM highest priority thread, associated with a $10 \mathrm{~Hz}$ timer, demands for control law computation. The platoon state is first updated: from vehicle kinematic model (1), the value of any variable in the platoon state is predicted at the current time instant, relying on a classical Runge-Kutta fourth-order method. These predictions, that are temporally consistent, can then be reported into lateral and longitudinal control laws, and the values supplied by these algorithms can be sent to the actuators. Of course, a watchdog has been considered: if some raw data have not been acquired (due to communication troubles for instance) for a long period (the threshold depends on vehicle velocities), then predictions are no longer computed and the vehicle begins a stopping phase. The deceleration is calculated according to the last acquired localization data of both the vehicle and its preceding one. If the communication trouble disappears, the vehicle is allowed to start again.

The variables within the platoon state that are the more sensitive to temporal inconsistencies are vehicle curvilinear distances $s_{i}$ along the reference trajectory. Fig. 4 presents the updates on these variables calculated by the third vehicle during a full-scale experiment. More precisely, the top figure shows the updates on the current (i.e. third) vehicle curvilinear distance, and the bottom one displays the updates on the leader curvilinear distance. It can be observed in the top figure that the update is non-negligible: it varies linearly, due to the quasi-constant difference between the control timer and the localization acquisition frequencies, and can reach $10 \mathrm{~cm}$ since 
the vehicle velocity and the control sampling frequency were respectively $1 \mathrm{~m} . \mathrm{s}^{-1}$ and $10 \mathrm{~Hz}$ (greater values are present at the beginning, because the vehicle is a bit faster than $1 \mathrm{~m} . \mathrm{s}^{-1}$ to reach the desired inter-vehicle distance). This illustrates the importance of AROCCAM task scheduling. The bottom figure shows that the update on variables obtained via communication channels is, of course, more significant: due to communication delays, it is generally superior to $4.7 \mathrm{~cm}$ (the distance, shown as a dotted line, covered by the vehicle during the mean communication delay $47 \mathrm{~ms}$ ) and can reach $30 \mathrm{~cm}$.

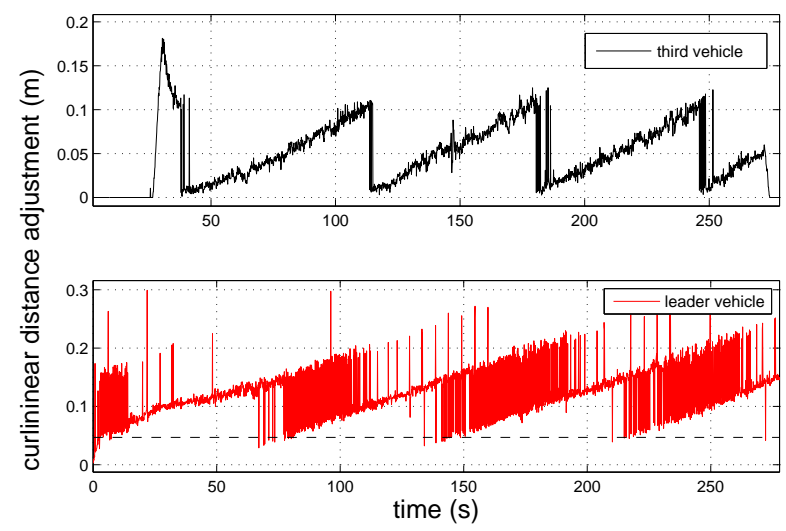

Fig. 4. Updates on curvilinear distance estimation

\section{OBSTACLE AVOIDANCE STRATEGY}

A key element in the proposed control approach is the reference trajectory. The error variables to be regulated in lateral and longitudinal control are either a gap w.r.t. the reference trajectory (i.e. $y_{i}$ and $\tilde{\theta}_{i}$ ) or a measurement w.r.t. this trajectory (i.e. $s_{i}$ ). As a result, the reference trajectory representation must exhibit the following features:

- it should accept an analytic expression, in order to enable an accurate computation of the error variables,

- it should be at least $C^{2}$, since the reference path curvature $c\left(s_{i}\right)$ is needed in control laws.

In order to meet these requirements, B-Spline curves have been used in previous work [21] and proved to be a relevant tool to address vehicle platooning. The B-Spline curves can be supplied a priori, in the case of a fully automated platoon, or created on-line according to the motion of a manually driven leader vehicle. Following [21], B-Spline curves of degree 3, with a $1.5 \mathrm{~m}$ arc-length for each polynomial curve, are here considered. Moreover, it is assumed that an accurate map of the environment, enclosing road sides, is available.

\section{A. Avoidance steps}

A lane change maneuver, combining circle and clothoids, is here proposed to carry out obstacle avoidance in a dedicated urban environment. The maneuver consists into 3 steps, shown in Fig. 5:

- When an obstacle is detected, it is first decided, relying on the environment map and on the obstacle features captured by the vehicle sensors (width, position, ....), if the obstacle can be safely avoided. If the answer is positive, then a virtual danger zone is defined around the obstacle as the smallest surrounding rectangle. At this step, this zone is only partially defined, since the obstacle depth is unknown. Nevertheless, two key locations can already be introduced: point $A$ is the projection of the vehicle controlled point on the nominal reference trajectory (i.e. $A$ is the current point $M$, defined in Fig. 2) and point $B$ is a waypoint on the road free space, sufficiently far from the obstacle, so that the vehicle can safely avoid this latter (here assumed to be motionless) if it moves to $B$. Then, an $S$ shaped curve, consisting in 4 clothoids and 2 optional circles, is defined to join $A$ to $B$, see Fig. 5. Clothoids are elementary curves characterized by a curvature $k(s)$ linearly varying with the arc-length:

$$
k(s)=\lambda s
$$

Since clothoids allow to reflect a natural driver's behaviour, they are the main element of the proposed $S$-shaped pattern. Circles are only introduced if the pattern built with 4 clothoids exhibits curvature values exceeding the vehicle admissible one.

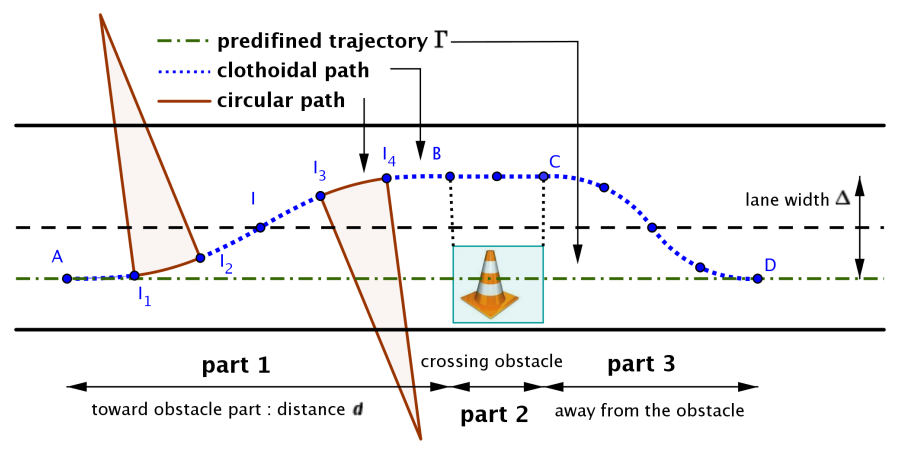

Fig. 5. Obstacle avoidance pattern

- When the vehicle is tracking the $S$-shaped pattern, it can eventually observe the obstacle length. Then, the third key location is defined: point $C$ is introduced in the road free space, sufficiently far from the obstacle, so that the vehicle can safely pass along this latter if it moves from $B$ to $C$. A $C$-shaped curve, consisting in only 2 clothoids, is defined to join $B$ and $C$. Such a pattern is appropriate in view of the curve profile of standard roads. If the obstacle is very long, then intermediary points can be considered to pass along it.

- Finally, the last key location is introduced: point $D$ is chosen on the nominal reference trajectory, in such a way that points $C$ and $D$ can be joined by 4 clothoids, see Fig. 5 .

\section{B. Pattern construction}

When building each pattern, the objective is to join two 2D locations, with the additional constraint to satisfy tangent and curvature continuity at these points. For instance, for the first pattern, the objective is to link the configuration $\left(A, q_{A}, k_{A}\right)$ to $\left(B, q_{B}, k_{B}\right)$, where $q_{\{A, B\}}$ and $k_{\{A, B\}}$ denote 
the tangent vector and the curvature at $A$ or $B$. In order to ensure $C^{2}$ continuity with the nominal reference path $\Gamma, q_{A}$ and $k_{A}$ are chosen as the tangent vector and curvature of $\Gamma$ at point $A$. In order to specify these variables at point $B$, let us introduce point $P$ as the closest point to $B$ on $\Gamma$. Then, $q_{B}$ is chosen identical to $q_{P}$, the tangent vector to $\Gamma$ at $P$, and $k_{B}=k_{P} /\left(\Delta k_{P}+1\right)$, with $\Delta$ the lane width and $k_{P}$ the curvature of $\Gamma$ at $P$.

The increasing curvature profile, induced by the first clothoid, allows to lead the avoidance trajectory towards the middle of the road, up to point $I_{1}$, see Fig. 5. The curvature $k_{I_{1}}$ at that point presents a local maximum. If $\left|k_{I_{1}}\right| \geq k_{\max }$, with $k_{\max }$ the vehicle admissible curvature $\left(k_{\max }\right.$ can be computed from the vehicle wheelbase $L$ and the maximum steering angle $\left.\delta_{\max }: k_{\max }=\left|\tan \left(\delta_{\max }\right) / L\right|\right)$, then a circle is introduced. The maximum curvature is then maintained, up to point $I_{2}$. The avoidance trajectory is still led towards the middle of the road. Next, an inflection point $I$ is reached (i.e. $k_{I}=0$ ) via the second clothoid. The same strategy is finally applied to join points $I$ and $B$. It can easily be noticed that this avoidance trajectory necessarily stays on the road and does not collide with the obstacle, as desired. Exactly the same method is followed to build the other patterns $B-C$ and $C-D$.

\section{Optimization procedure}

Let us consider the first $S$-shaped pattern linking $A$ to $B$ (once more, the same method has been applied to the other patterns). As a first step, a pattern consisting in only 4 clothoids is searched for. A clothoid starting from some given configuration is completely described when the rotation angle $\alpha$ (see Fig. 7) and the curvature at its extremity are both given. Consequently, since a $S$-shaped pattern encloses an inflexion point $\left(k_{I}=0\right)$, then it is completely described by 6 parameters: $\left(\alpha_{1}, \alpha_{2} \beta_{1}, \beta_{2}, k_{I_{1}}\right.$ and $\left.k_{I_{3}}\right)$, see Fig. 7. In order to simultaneously reduce the number of parameters and provide a "balanced" avoidance trajectory, the curvature rates of the 4 clothoids are chosen identical (i.e. the parameter $\lambda$ in (2) is the same for the 4 clothoids). Then, the $S$-shaped pattern is completely specified by only 2 parameters: $\alpha_{1}$ and $k_{I_{1}}$. Parameter $k_{I_{1}}$ mainly acts on the longitudinal aspect of the $S$-shaped curve (i.e. in the direction of the vector $\overrightarrow{A B}$ ), when $\alpha_{1}$ value impacts its lateral aspect (i.e. in the direction perpendicular to the vector $\overrightarrow{A B}$ ).

The parameters $\left(\alpha_{1}, k_{I_{1}}\right)$ of the $S$-shaped pattern linking $A$ to $B$ could be computed analytically via Fresnel integrals. However, calculations are quite intricate and time-consuming. Consequently, the concept of discrete clothoid, developed in [14] and shown in Fig. 6, has here been used. A discrete clothoid of arc-length $S$ is formed of $n+1$ arcs, numbered 0 to $n$ (with $n \geq 1$ ). Arc 0 and arc $n$ have length $S /(2 n)$, while arcs 1 to $n-1$ have length $S / n$. The curvatures of the $n+1$ arcs are $k_{j}=k_{0}+j h, j=0,1 \ldots, n$, where here $h=\left(k_{B}-k_{A}\right) / n$. It is proved [14] that the approximation error between the exact clothoid and a discrete clothoid with $n+1$ arcs is of magnitude $O\left((1 / n)^{2}\right)$.

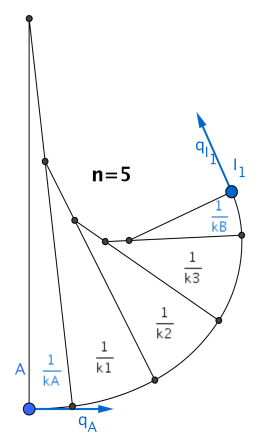

Fig. 6. Discrete clothoid representation

When beginning the optimization procedure, $n$ is chosen low, in order to quickly obtain a rough estimation of the $S$ shaped pattern. Next, $n$ is increased, in order to refine the pattern. If $\left|k_{I_{1}}\right|$ or $\left|k_{I_{3}}\right|$ exceed $k_{\max }$, circles are added to the $S$-shaped pattern by introducing two new parameters $\omega_{1}$ and $\omega_{2}$, defined as the rotation angles of the circles, see Fig. 7. Then, the same final configuration, but with admissible curvature values, can be obtained.

Finally, in order to be consistent with the nominal reference trajectory $\Gamma$ defined with $B$-Spline curves, the alternative one, constituted of $S / C$-shaped trajectories, is also approximated thanks to $B$-Spline curves. The approximation is carried out via standard least square method. However, a special attention is paid to the curvature profile: first, the optimization criterion aims at minimizing both distances and tangent angle deviations between a configuration set extracted on the $S / C$-shaped trajectories and the approximating $B$-Spline curve. Then, since B-Spline curves have not been designed to present a linear relation between arc-length and curvature, the arc-length of elementary polynomial curves has to be chosen shorter than in the nominal case: $0.5 m$-long polynomial curves are considered, rather than $1.5 m$-long ones for the nominal trajectory $\Gamma$. The curvature profile of the $B$-Spline approximating the alternative trajectory is then very similar to the desired one (maximum curvature error equals to 0.0281 ), thus providing a comfortable guidance.

\section{EXPERIMENTAL RESULTS}

In order to investigate the capabilities of the proposed approach, preliminary experiments have been carried out with three vehicles in Clermont-Ferrand on "PAVIN Site" (see Fig. 1), an open platform devoted to urban transportation system evaluation.

1) Experimental set-up: Experimental vehicles are shown in Fig. 1. They are electric vehicles, powered by lead-acid batteries providing 2 hours autonomy. Two (resp. four) passengers can travel aboard Cycabs (resp. RobuCabs). Their small dimensions (length $1.90 \mathrm{~m}$, width $1.20 \mathrm{~m}$ ) and their maximum speed $\left(5 \mathrm{~m} . \mathrm{s}^{-1}\right)$ are appropriate to urban environments. On-board RTK GPS receivers provide absolute localization 
measurements accurate to within $2 \mathrm{~cm}$ at a $10 \mathrm{~Hz}$ sampling frequency. Platoon control laws are implemented in $\mathrm{C}++$ language on Pentium-based computers using RTAI-Linux OS.

2) Experimental results: The behaviour of an automatic guided platoon involving two vehicles has been investigated. A motionless third vehicle (green rectangle in Fig. 8) is obstructing the $100 \mathrm{~m}$-long reference path at arc-length abscissa $55 m$ (see also Fig. 1).

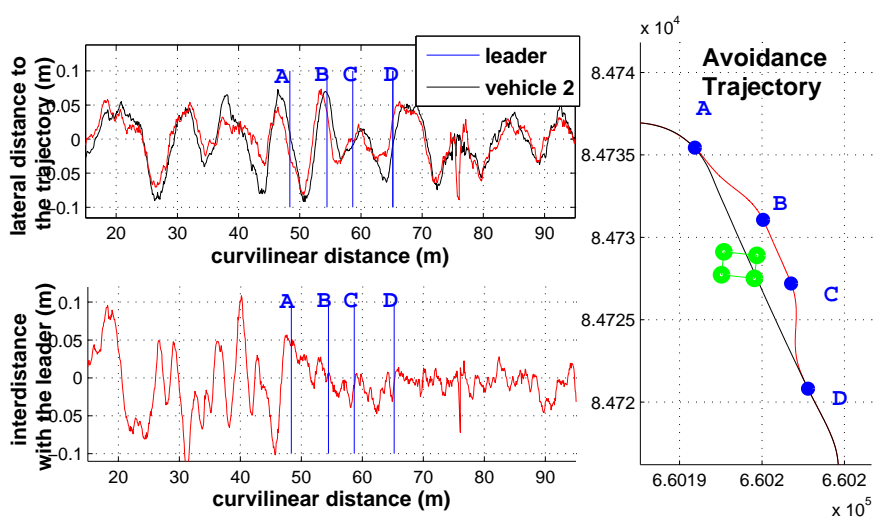

Fig. 8. Vehicle lateral and inter-distance errors

The leader vehicle velocity is $1 \mathrm{~m} . \mathrm{s}^{-1}$. and the obstacle is perceived during a bend $\left(k_{A}=-0.2589\right)$. At that time, the curvilinear distance between the leader and the obstacle is $7.0193 \mathrm{~m}$. The leader vehicle successfully computes an alternative admissible trajectory, whose maximum curvature is $0.2604 m^{-1}$ (when $k_{\max }=0.3886 \mathrm{~m}^{-1}$ ). This alternative trajectory is then sent to the follower vehicle, so that both vehicles manage to avoid the obstacle. Moreover, the accuracy of the lateral and longitudinal control laws is preserved. As illustrated in Fig. 8, the behavior is identical to what was obtained in previous work, when vehicles were guided w.r.t. a fixed and pre-specified reference trajectory (see [7]), namely a $10 \mathrm{~cm}$ accuracy. The on-line reference trajectory modification disturbs neither lateral nor longitudinal performances.

\section{CONCLUSION}

In this paper, communication issues within a platoon of vehicles have first been addressed. A data actualization procedure has been proposed in order to ensure temporal consistence when implementing the global decentralized control strategy. Then, an on-line reference trajectory modification, consisting in reshaping the B-Spline curves used to define the nominal reference path, has been developed in order to address obstacle avoidance: the alternative trajectory approximates the shape and the curvature profile of clothoids, in order to reflect a natural driver's behaviour. Finally, full scale experiments, carried out with three vehicles, have demonstrated the efficiency of the proposed approach.

\section{REFERENCES}

[1] S. Benhimane and E. Malis. Vision-based control for car platooning using homography decomposition. In IEEE International Conference on Robotics and Automation (ICRA), pages 2173-2178, 2005.
[2] D. Wang and M. Pham. Unified control design for autonomous carlike vehicle tracking maneuvers, chapter 8 in "Autonomous Mobile Robots: sensing, control decision-making and applications", pages 295329. CRC Press, May 2006.

[3] L Consolini, F Morbidi, D Prattichizzo, and M Tosques. Leader-follower formation control of nonholonomic mobile robots with input constraints. Automatica, 2008.

[4] Soo-Yeong Yi and Kil-To Chong. Impedance control for a vehicle platoon system. Mechatronics, 2004.

[5] Rafael E Caicedo, John Valasek, and John L Junkins. Preliminary results of one-dimensional vehicle formation control using structural analogy. In American Control Conference, 2003.

[6] Wenjie Dong and Yi Guo. Formation Control of Nonholonomic Mobile Robots Using Graph Theoretical Methods, Springer-Verlag, chapter Cooperative Systems, pages 369-386. 2007.

[7] J. Bom, B. Thuilot, F. Marmoiton, and P. Martinet. Nonlinear control for urban vehicles platooning, relying upon a unique kinematic GPS. In $22^{\text {nd }}$ Intern. Conf. on Robotics and Automation (ICRA'05), Barcelona (Spain), April 2005.

[8] D. Swaroop and Seok Min Yoon. The design of a controller for a following vehicle in an emergency lane change maneuver. California PATH Working paper, 1999.

[9] P. Jacobs and J. Canny. Planning smooth paths for mobile robots. In IEEE Int. Conf. Robotics and Automation, volume 1, pages 2-7, 1989.

[10] J.P. Laumond, J Jacobs, M Taix, and M.R. Murray. A motion planner for nonholonomic mobile robots. IEEE Trans. Robotics \& Automation, 10:577-593, 1994.

[11] T. Fraichard, A. Scheuer, and R. Desvigne. From Reeds and Sheeps to continuous curvature paths. In IEEE Int. Conf. on Advanced Robotics, pages 585-590, 1999.

[12] A. Scheuer and T. Fraichard. Collision-free and continuous curvature path planning for car-like robots. In IEEE Int. Conf. Robotics and Automation, 1997.

[13] L.Z. Wang, K.T. Miura, E. Nakamae, T. Yamamoto, and T.J Wang. An approximation approach of the clothoid curve defined in the interval $[0, \pi / 2]$ and its offset by free-form curves. Computer Aided Design, 33(14):1049-1058, 2001.

[14] D.S. Meek and D.J. Walton. An arc spline approximation to a clothoid. J. of Comp.l and App. Math., 170:59-77, 2004.

[15] J. Sànchez-Reyes and J.M. Chacón. Polynomial approximation to clothoids via s-power series. Computer Aided Design, 35(14):13051319, 2003.

[16] N Montés, Marta C. Mora, and Josep Tornero. Trajectory generation based on rational bezier curves as clothoids. IEEE Intelligent Vehicles Symposium, 2007.

[17] C. Samson. Control of chained systems: application to path following and time-varying point stabilization of mobile robots. IEEE Trans. on Automatic Control, 40(1):64-77, January 1995.

[18] B. Thuilot, J. Bom, F. Marmoiton, and P. Martinet. Accurate automatic guidance of an urban electric vehicle relying on a kinematic GPS sensor. In $5^{\text {th }}$ IFAC Symposium on Intelligent Autonomous Vehicles (IAV'O4), Lisboa (Portugal), July 2004.

[19] E. Royer, M. Lhuillier, M. Dhome, and T. Chateau. Towards an alternative GPS sensor in dense urban environment from visual memory. In British Machine Vision Conference, volume 1, pages 197-206, Kingston (England), September 2004.

[20] C. Tessier, C. Cariou, C. Debain, R. Chapuis, F. Chausse, and C. Rousset. A real-time, multi-sensor architecture for fusion of delayed observations: Application to vehicle localization. In $9^{\text {th }}$ Intern. IEEE Conference on Intelligent Transportation Systems, ITSC 2006, pages 1316-1321, Toronto (Canada), Sept. 2006.

[21] P. Avanzini, B. Thuilot, T. Dallej, P. Martinet, and J-P. Derutin. Online reference trajectory generation for manually convoying a platoon of automatic urban vehicles. In Intern. Conf. on Intelligent Robots and Systems (IROS'09)), Saint-Louis (USA), October 2009. 